\title{
Dynamic analysis of geared robotic mechanisms by the concept of torque transmission
}

\author{
Dar-Zen Chen*, ${ }^{*}$, Ta-Wei Lin, Yi-Liang Lin \\ Department of Mechanical Engineering, National Taiwan University, Taipei 106, Taiwan
}

Received 8 July 1998; accepted 5 May 1999

\begin{abstract}
An efficient two-step methodology for the inverse dynamics of geared robotic mechanisms (GRMs) is developed. In the first step, with the effective external forces, input transmitted forces are computed from the moment balance equation along axes of rotation starting from the end primary link backward along the heavy-edged path in a mechanical transmission line (MTL). The computation begins from the highest level MTL towards the lowest level MTL, one or two level at a time depending on the topological structure of the GRM. It is shown that the dynamic equations can be efficiently established from the computed input transmitted forces. In the second step, the remaining unsolved reaction forces are evaluated link-by-link backward along the heavy-edged paths from the highest level MTL to the lower level ones. Thus, the inverse dynamic problem can be solved efficiently and systematically without solving the entire system of equations simultaneously. (C) 1999 Elsevier Science Ltd. All rights reserved.
\end{abstract}

Keywords: Dynamic analysis; Geared robotic mechanism; Gear contact force

\section{Introduction}

Many robot manipulators take the form of partially closed-loop configuration to reduce the mass and inertia loads of actuators. For geared robotic mechanisms (GRMs), gear trains are introduced so that actuators can be located as close to the base as possible. Many generalpurpose computer programs, such as ADAMS [1] and DADS [2] are capable of modeling the

\footnotetext{
* Corresponding author. Tel.: + 886-2362-1522; fax: + 886-2763-1755.

E-mail address: dzchen@ccms.ntu.edu.tw (D.-Z. Chen).

${ }^{1}$ Paper No. MMT 33-66.

0094-114X/00/\$ - see front matter (C) 1999 Elsevier Science Ltd. All rights reserved.

PII: S0094-114X(99)00041-5
} 
kinematics and dynamics of many mechanical systems. However, they often require usersupplied subroutines to model complicated mechanical systems, such as planetary gear trains with floating carrier. The efficient methodologies, such as the iterative Lagrange-Euler approach [3], recursive Lagrange approach [4], recursive Newton-Euler approach [5-8], and the generalized d'Alembert approach [9] are tailored for the dynamic analysis of open-loop manipulators. However, these studies did not address the coupling effects of the gears and rotors although most robot manipulators are driven by motors through gear trains. Strictly speaking, these formulations are only applicable to direct-drive type manipulators. Tsai et al. [10] developed a systematic "level-by-level" method for the dynamic analysis of GRMs. In their method, links are classified into levels according to the length of thin-edged paths from the base, and reaction forces are evaluated link-by-link from the highest level towards the base. Dynamic loads on bearings and gear teeth are evaluated by the balance equations of each link and dynamic equations are formed accordingly. However, there are five or six unknowns in the link's balance equations to be determined at a time, and dynamic equations can be formed only when all the dynamic loads are evaluated.

In this paper, an efficient and systematic two-step procedure for the formulation of dynamic equations and for the evaluation of dynamic loads of GRMs will be presented. The approach modifies the Newton-Euler recursive inverse dynamics algorithm for the open-loop chains and tree structures to include gear joints. It will be shown that input transmitted forces of links can be evaluated efficiently and systematically from the highest level mechanical transmission line (MTL) towards the lowest level MTL, one or two level at a time depending on the topological structure of the mechanism. It will also be shown that only the input transmitted forces and effective external forces of each link are necessary to be evaluated to form the system's dynamic equations. With the evaluated input transmitted forces and computed effective external forces, remaining unknown reaction forces can be determined from the balance

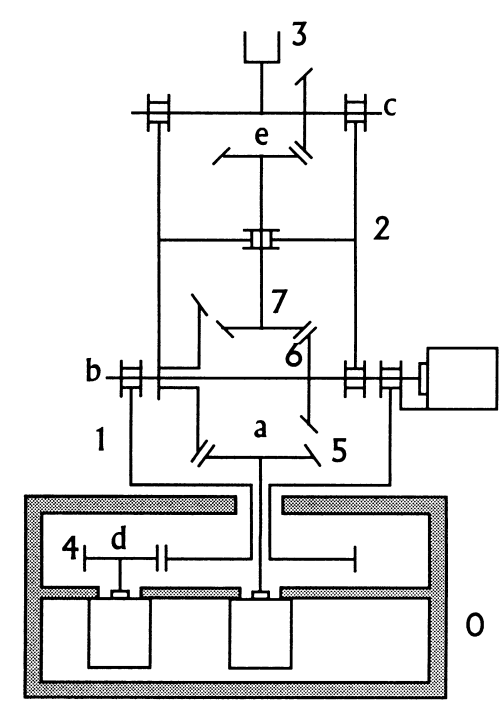

Fig. 1. A 3-dof GRM. 
equation of links. This procedure is especially useful when the evaluation of dynamic loads of bearings and gear teeth is desired for the design of such mechanisms. The 3 degree-of-freedom (dof) GRM shown in Fig. 1 will be used as an illustrative example.

\section{Graphical representation}

In the graphical representation, links are denoted by vertices, turning pairs by thin edges,

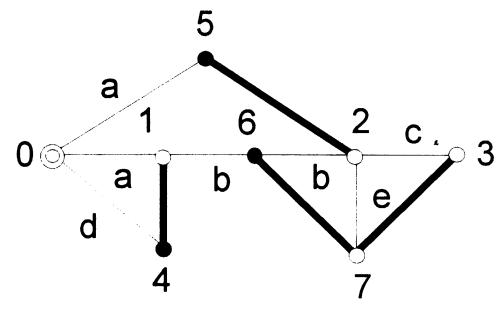

(a)

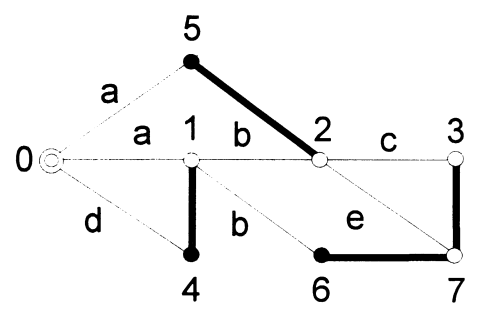

(b)

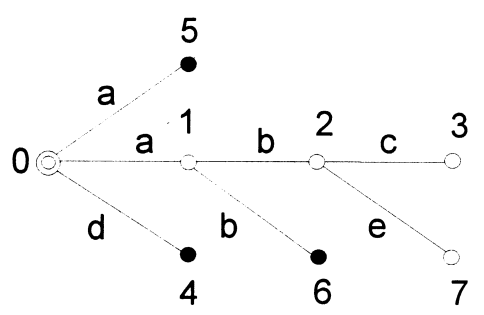

(c)

Fig. 2. (a) Graph representation. (b) Canonical graph. (c) Revolute-joint-only spanning tree. 
gear pairs by heavy edges and thin edges are labeled according to their axes locations. Fig. 2(a) shows the graphical representation of the example GRM. A unique canonical graph [11] can be obtained through edge re-configuration of the graphical representation so that all edges lying on a thin-edged path traced from the base to any other vertex have different edge labels. Fig. 2(b) shows the canonical graph of the example 3-dof GRM. From the canonical graph, a thinedged-only spanning tree, shown in Fig. 2(c), can be obtained by removing all the heavy edges. In the spanning tree, each thin-edged path originating from the base and ending at a leaf [12] represents an open-loop chain. Among these open-loop chains, the one that starts from the base and ends at the output link is defined as the equivalent open-loop chain (EOLC) [11]. Each link in the EOLC is referred to as a primary link, while all other links are called secondary links [13]. Note that the last link of EOLC is a primary link, while the last links of other open-loop chains are secondary links. A secondary link is called an input link if it is attached to an actuator. A secondary link $j$ is said to be carried by a primary link $i$ if it is connected to primary link $i$ with a thin-edge in the canonical graph. The revolute joint that connects two primary links is called a primary joint, while the revolute joint that connects a primary link and a secondary link is a secondary joint. Primary joint angle $\theta_{i, i-1}$ is the angular displacement of primary link $i$ with respect to primary link $(i-1)$ and secondary joint angle $q_{j, i}$ is the angular displacement of secondary link $j$ with respect to primary link $i$ that carries it. From Fig. 2(b), it can be seen that thin-edged path 0-1-2-3 is the EOLC. Links 0, 1, 2, and 3 are primary links, while links 4, 5, 6, and 7 are secondary links. Primary link 3 is the end effector. Secondary links 4 and 5 are carried by primary link 0 , secondary link 6 is carried by primary link 1, and secondary link 7 is carried by primary link 2 .

\section{Kinematics of links}

\subsection{Primary links}

Beginning from the base link, primary links are numbered sequentially from 0 to $n$. For primary link $i$, a coordinate system $\left(\boldsymbol{x}_{i}, \boldsymbol{y}_{i}, \boldsymbol{z}_{i}\right)$ is attached to the distal end of link $i$ in accordance with the D-H convention [14], where $d_{i, i-1}$ is the translational distance along $z_{i-1}{ }^{-}$ axis, $a_{i, i-1}$ and $\alpha_{i, i-1}$ are the offset distance and twist angle between $z_{i-1}$-axis and $z_{i}$-axis, respectively. Note that the angle measured from $\boldsymbol{x}_{i-1}$-axis to $\boldsymbol{x}_{i}$-axis about $\boldsymbol{z}_{i-1}$-axis, $\theta_{i, i-1}$, is the primary joint angle. The spatial relationship between the $i$ th and $(i-1)$ th coordinate systems can be described by a rotation matrix as:

$$
{ }^{i-1} \boldsymbol{R}_{i}=\left[\begin{array}{lll}
C \theta_{i, i-1} & -C \alpha_{i, i-1} S \theta_{i, i-1} & S \alpha_{i, i-1} S \theta_{i, i-1} \\
S \theta_{i, i-1} & C \alpha_{i, i-1} C \theta_{i, i-1} & -S \alpha_{i, i-1} C \theta_{i, i-1} \\
0 & S \alpha_{i, i-1} & C \alpha_{i, i-1}
\end{array}\right]
$$

where $C \theta_{i}, i-1=\cos \left(\theta_{i}, i-1\right), S \theta_{i}, i-1=\sin \left(\theta_{i}, i-1\right), C \alpha_{i}, i-1=\cos \left(\alpha_{i}, i-1\right)$, and $S \alpha_{i}, i-1$ $=\sin \left(\alpha_{i}, i-1\right)$.

The kinematic properties of primary links can be computed from the first moving link towards the end-effector by the forward recursive equations [8]: 


$$
\begin{aligned}
& { }^{i} \boldsymbol{\omega}_{i}={ }^{i} \boldsymbol{R}_{i-1}\left({ }^{i=1} \boldsymbol{\omega}_{i-1}+\dot{\theta}_{i, i-1}{ }^{i-1} \boldsymbol{z}_{i-1}\right) \\
& { }^{i} \dot{\boldsymbol{\omega}}_{i}={ }^{i} \boldsymbol{R}_{i-1}\left({ }^{i-1} \dot{\boldsymbol{\omega}}_{i-1}+\ddot{\theta}_{i, i-1}{ }^{i-1} \boldsymbol{z}_{i-1}+{ }^{i-1} \boldsymbol{\omega}_{i-1} \times \dot{\theta}_{i, i-1}{ }^{i-1} \boldsymbol{z}_{i-1}\right) \\
& { }^{i} \dot{\boldsymbol{v}}_{i}={ }^{i} \dot{\boldsymbol{\omega}}_{i} \times{ }^{i} \boldsymbol{p}_{i, i-1}+{ }^{i} \boldsymbol{R}_{i-1}{ }^{i-1} \dot{\boldsymbol{v}}_{i-1}+{ }^{i} \boldsymbol{\omega}_{i} \times\left({ }^{i} \boldsymbol{\omega}_{i} \times{ }^{i} \boldsymbol{p}_{i, i-1}\right)
\end{aligned}
$$

and

$$
{ }^{i} \dot{\boldsymbol{v}}_{i c}={ }^{i} \dot{\boldsymbol{\omega}}_{i} \times{ }^{i} \boldsymbol{r}_{i c}+{ }^{i} \dot{\boldsymbol{v}}_{i}+{ }^{i} \boldsymbol{\omega}_{i} \times\left({ }^{i} \boldsymbol{\omega}_{i} \times{ }^{i} \boldsymbol{r}_{i c}\right)
$$

where $\boldsymbol{p}_{i, i-1}$, the position vector defined from $o_{i-1}$ to $o_{i}$, can be written as

$$
{ }^{i} \boldsymbol{p}_{i, i-1}=\left[\boldsymbol{a}_{i, i-1}, \boldsymbol{d}_{i, i-1} \boldsymbol{S} \alpha_{i, i-1}, \boldsymbol{d}_{i, i-1} \boldsymbol{C} \alpha_{i, i-1}\right]^{\mathrm{T}}
$$

Fig. 3 shows the coordinate systems of the primary links and $o_{i}$ is the origin of $\left(\boldsymbol{x}_{i}, \boldsymbol{y}_{i}, \boldsymbol{z}_{i}\right)$ coordinate system. From Fig. 3 , we have $a_{1}, 0=a_{3}, 2=d_{2}, 1=d_{3}, 2=\alpha_{2}, 1=0, \alpha_{1}, 0=\alpha_{3}, 2=$ $\pi / 2, a_{2,1}=a_{21}$ and $d_{1,0}=d_{10}$. Thus, from Eq. (6), we have

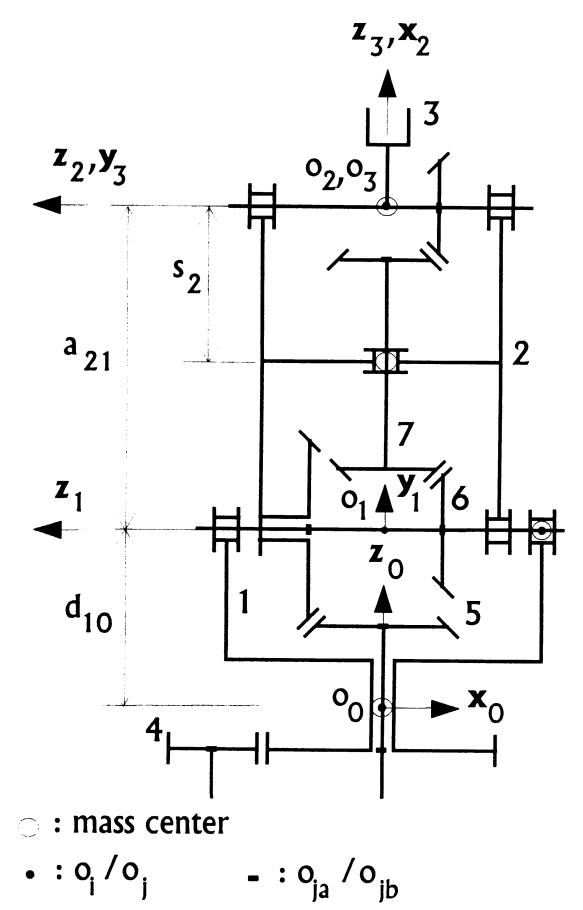

Fig. 3. Coordinate systems of primary links. 
${ }^{1} \boldsymbol{p}_{1,0}=\left[0, d_{10}, 0\right]^{\mathrm{T}}, \quad{ }^{1} \boldsymbol{p}_{2,1}=\left[a_{21}, 0,0\right]^{\mathrm{T}}, \quad$ and $\quad{ }^{1} \boldsymbol{p}_{3,2}=[0,0,0]^{\mathrm{T}}$

\subsection{Secondary links}

For a secondary link $j$ carried by primary link $i$, a coordinate system $\left(\boldsymbol{x}_{j}, \boldsymbol{y}_{j}, \boldsymbol{z}_{j}\right)$ can also be established in accordance with the $\mathrm{D}-\mathrm{H}$ convention. Note that the angle measured from $\boldsymbol{x}_{i}$ to $\boldsymbol{x}_{j}$ along $\boldsymbol{z}_{i}$-axis, $\theta_{j, i}$, is constant since the $\left(\boldsymbol{x}_{j}, \boldsymbol{y}_{j}, \boldsymbol{z}_{j}\right)$ coordinate system is fixed in the $\left(\boldsymbol{x}_{i}, \boldsymbol{y}_{i}, \boldsymbol{z}_{i}\right)$ coordinate system. The transformation relationship from the $\left(\boldsymbol{x}_{j}, \boldsymbol{y}_{j}, \boldsymbol{z}_{j}\right)$ to the $\left(\boldsymbol{x}_{i}, \boldsymbol{y}_{i}, \boldsymbol{z}_{i}\right)$ coordinate systems can be established by the rotation matrix as:

$$
{ }^{i} \boldsymbol{R}_{j}=\left[\begin{array}{lll}
C \theta_{j, i} & -C \alpha_{j, i} S \theta_{j, i} & S \alpha_{j, i} S \theta_{j, i} \\
S \theta_{j, i} & C \alpha_{j, i} C \theta_{j, i} & -S \alpha_{j, i} C \theta_{j, i} \\
0 & S \alpha_{j, i} & C \alpha_{j, i}
\end{array}\right]
$$

The angular displacement of secondary link $j$ with respect to primary link $i$ that carries it, $q_{j, i}$, can be expressed in terms of primary joint angles by using the fundamental circuit theory and coaxial conditions [11]. The vector of secondary joint angles of input links, $\boldsymbol{Q}$, can be derived as a function of the vector of primary joint angles, $\Theta$, as:

$$
[Q]=A^{\mathrm{T}}[\Theta]
$$

where $\boldsymbol{A}$ is the structure matrix [15].

Chang and Tsai [15] showed that the series of non-zero elements in each column of structure matrix $\boldsymbol{A}$ represent an MTL. The ith column of the structure matrix depicts the constituents of the $i$ th MTL. Accordingly, associated vertices and edges in the canonical graph representation can be differentiated so that the sub-graphs representing the MTLs are identified. Therefore, a GRM can be decomposed into MTLs that represent different paths to transmit the input torques. An MTL is called MTL of level $i$ if its heavy-edged path ends at primary link $i$. A structure matrix is said to be of pseudo-triangular form if each primary link is the end link of an MTL. The length of an MTL is defined as the number of articulated joints in it. Note that the input link contains only output-end gear, while other secondary links contain both inputend and output-end gear and the end primary link contains only input-end gear.

Since secondary link $j$ rotates along $z_{j}$-axis with respect to primary link $i$, the angular velocity of secondary link $j$ can be computed by modifying Eq. (2) with the secondary joint angle $q_{j, i}$ as:

$$
{ }^{j} \boldsymbol{\omega}_{j}={ }^{j} \boldsymbol{R}_{i}{ }^{i} \boldsymbol{\omega}_{i}+\dot{q}_{j, i}{ }^{j} \boldsymbol{z}_{j}
$$

Assuming all secondary links are symmetric about their rotational axes, mass center of secondary link $j$ is a fixed point with respect to the primary link, which carries it. Hence, the mass center velocity of secondary link $j$ can be derived by modifying Eq. (5) as:

$$
{ }^{j} \dot{\boldsymbol{v}}_{j c}={ }^{j} \boldsymbol{R}_{i}^{i} \dot{\boldsymbol{\omega}}_{i} \times\left({ }^{j} \boldsymbol{p}_{j, i}+{ }^{j} \boldsymbol{r}_{j c}\right)+{ }^{j} \boldsymbol{R}_{i}^{i} \dot{\boldsymbol{v}}_{i}+{ }^{j} \boldsymbol{R}_{i}^{i} \boldsymbol{\omega}_{i} \times\left[{ }^{j} \boldsymbol{R}_{i}^{i} \boldsymbol{\omega}_{i} \times\left({ }^{j} \boldsymbol{p}_{j, i}+{ }^{j} \boldsymbol{r}_{j c}\right)\right]
$$

where $\boldsymbol{p}_{j, i}$, the position vector defined from $o_{i}$ to $o_{j}$, can be written as: 


$$
{ }^{j} \boldsymbol{p}_{j, i}=\left[a_{j, i}, d_{j, i} S \alpha_{j, i}, d_{j, i} C \alpha_{j, i}\right]^{\mathrm{T}}
$$

Fig. 4 shows the coordinate systems of secondary links of the example GRM. From Fig. 4, we have $a_{5}, 0=a_{6}, 1=a_{7}, 2=d_{5}, 0=d_{7}, 2=\alpha_{4}, 0=\alpha_{5}, 0=\alpha_{6}, 1=\theta_{4}, 0=\theta_{5}, 0=0, \alpha_{7}, 2=\theta_{6}, 1=\theta_{7,2}=$ $\pi / 2, a_{4,0}=-a_{40}, d_{4,0}=-d_{40}$, and $d_{6,1}=-d_{61}$. Thus, from Eq. (12), we have

$$
\begin{aligned}
& { }^{4} \boldsymbol{p}_{4,0}=\left[-a_{40}, 0,-d_{40}\right]^{\mathrm{T}}, \quad{ }^{5} \boldsymbol{p}_{5,0}=[0,0,0]^{\mathrm{T}}, \quad{ }^{6} \boldsymbol{p}_{6,1}=\left[0,0,-d_{61}\right]^{\mathrm{T}}, \quad \text { and } \\
& { }^{7} \boldsymbol{p}_{7,2}=[0,0,0]^{\mathrm{T}}
\end{aligned}
$$

From Fig. 2(b), angular displacements $\theta_{1}, 0, \theta_{2}, 1$, and $\theta_{3,2}$ are primary joint angles, while $q_{4}$, $0, q_{5}, 0, q_{6}, 1$ and $q_{7,2}$ are secondary joint angles. Since links 4,5 , and 6 are the input links, we have $[Q]=\left[q_{4}, 0, q_{5}, 0, q_{6}, 1\right]^{\mathrm{T}},[\Theta]=\left[\theta_{1}, 0, \theta_{2}, 1, \theta_{3}, 2\right]^{\mathrm{T}}$, and the structure matrix can be shown as:

$$
\boldsymbol{A}=\left[\begin{array}{lll}
e_{14} & 1 & 0 \\
0 & e_{25} & 1 \\
0 & 0 & e_{37} e_{76}
\end{array}\right]
$$

where $e_{k j}= \pm n_{k} / n_{j}$ is the gear ratio, positive or negative depends on a positive rotation of link

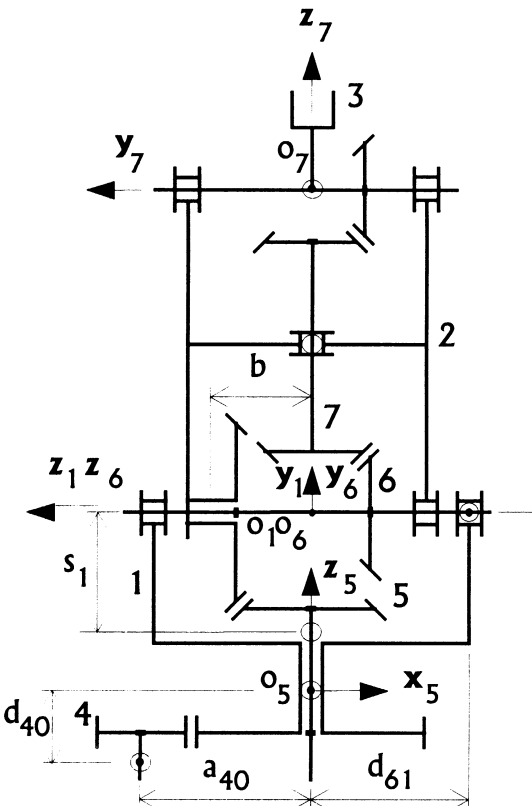

$$
\begin{aligned}
& \text { : mass center } \\
& -: o_{i} / o_{j} \quad-: o_{j a} / o_{j b}
\end{aligned}
$$

Fig. 4. Coordinate systems of secondary links. 
$k$ resulting in a positive or negative rotation of link $j$, and $n_{k}$ and $n_{j}$ are the teeth numbers of gear mounted on link $k$ and link $j$, respectively.

\section{Forces representation}

\subsection{Inertia force}

With the known kinematic properties of links, the inertia force $\boldsymbol{F}_{i}^{*}$ and moment $\boldsymbol{N}_{i}^{*}$ of a link $i$ can be evaluated by:

$$
\boldsymbol{F}_{i}^{*}=m_{i} \dot{\boldsymbol{v}}_{i c}
$$

and

$$
\boldsymbol{N}_{i}^{*}={ }^{c} \boldsymbol{I}_{i} \dot{\boldsymbol{\omega}}_{i}+\boldsymbol{\omega}_{i} \times\left({ }^{c} \boldsymbol{I}_{i} \boldsymbol{\omega}_{i}\right)
$$

where $\dot{\boldsymbol{v}}_{i c}$ is the linear acceleration of mass center of link $i$ and ${ }^{c} \boldsymbol{I}_{i}$ is the inertia tensor of link $i$ with respect to its mass center.

\subsection{Reaction forces at revolute joints}

The reaction force exerted by link $(i-1)$ to link $i$ at revolute joint $(i, i-1), f_{i, i-1}$, can be expressed in the $\left(\boldsymbol{x}_{i}, \boldsymbol{y}_{i}, \boldsymbol{z}_{i}\right)$ coordinate system as:

$$
\left.\left.\left.{ }^{i} \boldsymbol{f}_{i, i-1}=\left[\boldsymbol{f}_{i, i-1}\right)_{x},{ }^{i} \boldsymbol{f}_{i, i-1}\right)_{y},{ }^{i} \boldsymbol{f}_{i, i-1}\right)_{z}\right]^{\mathrm{T}}
$$

Since a revolute joint cannot transmit torques about its joint axis for the absence of friction, the reaction moment exerted by link $(i-1)$ to link $i$ at revolute joint $(i, i-1), \boldsymbol{n}_{i, i-1}$, can be expressed in the $\left(\boldsymbol{x}_{i}, \boldsymbol{y}_{i}, \boldsymbol{z}_{i}\right)$ coordinate system as:

$$
\left.\left.{ }^{i} \boldsymbol{n}_{i, i-1}=\left[{ }^{i} \boldsymbol{n}_{i, i-1}\right)_{x}, \boldsymbol{n}_{i, i-1}\right)_{y}, 0\right]^{\mathrm{T}}
$$

For the case that link $i$ is an input link, Eq. (18) becomes:

$$
\left.\left.{ }^{i} \boldsymbol{n}_{i, i-1}=\left[{ }^{i} \boldsymbol{n}_{i, i-1}\right)_{x}, \boldsymbol{n}_{i, i-1}\right)_{y}, \xi\right]^{\mathrm{T}}
$$

where $\xi$ is the input torque.

\subsection{Gear contact forces}

Assuming the output-end gear of secondary link $j$ mesh with the input-end gear of secondary link $k$, and primary link $i$ be the carrier of the gear pair. Let $\boldsymbol{x}_{j b}$ be a unit vector pointing from the center of the output-end gear, $o_{j b}$, to the contact point of the meshed gear pair, $z_{j b}$-axis is a unit vector along $z$ j-axis, and $\boldsymbol{y}_{j b}$ is determined by the right-hand rule. Then, a local output-end 
coordinate system, $\left(\boldsymbol{x}_{j b}, \boldsymbol{y}_{j b}, \boldsymbol{z}_{j b}\right)$, can be established. Since the contact point of a gear pair is fixed with respect to its carrier, the $\left(\boldsymbol{x}_{j b}, \boldsymbol{y}_{j b}, \boldsymbol{z}_{j b}\right)$ coordinate system is fixed in the $\left(\boldsymbol{x}_{i}, \boldsymbol{y}_{i}, \boldsymbol{z}_{i}\right)$ coordinate system and the transformation matrix from the $\left(\boldsymbol{x}_{j b}, \boldsymbol{y}_{j b}, \boldsymbol{z}_{j b}\right)$ to the $\left(\boldsymbol{x}_{i}, \boldsymbol{y}_{i,}, \boldsymbol{z}_{i}\right)$ coordinate systems can be described as:

$$
{ }^{i} \boldsymbol{H}_{j b}=\left[\begin{array}{lll}
\boldsymbol{x}_{i} \cdot \boldsymbol{x}_{j b} & \boldsymbol{x}_{i} \cdot \boldsymbol{y}_{j b} & \boldsymbol{x}_{i} \cdot \boldsymbol{z}_{j b} \\
\boldsymbol{y}_{i} \cdot \boldsymbol{x}_{j b} & \boldsymbol{y}_{i} \cdot \boldsymbol{y}_{j b} & \boldsymbol{y}_{i} \cdot \boldsymbol{z}_{j b} \\
\boldsymbol{z}_{i} \cdot \boldsymbol{x}_{j b} & \boldsymbol{z}_{i} \cdot \boldsymbol{y}_{j b} & \boldsymbol{z}_{i} \cdot \boldsymbol{z}_{j b}
\end{array}\right]
$$

Similarly, a local input-end coordinate system, $\left(\boldsymbol{x}_{j a}, \boldsymbol{y}_{j a}, \boldsymbol{z}_{j a}\right)$, can also be set up on the inputend gear of link $j$. Also, the transformation matrix from the $\left(\boldsymbol{x}_{j a}, \boldsymbol{y}_{j a}, \boldsymbol{z}_{j a}\right)$ coordinate system to the coordinate system of its associated carrier can be defined. Note that only the output-end coordinate system can be defined for an input link, while only the input-end coordinate system can be defined for a primary link which has gear mesh with a secondary link.

The gear contact force exerted by the input-end gear of a secondary link $k$ to the output-end gear of a secondary link $j \boldsymbol{g}_{j, k}$, can be represented in the $\left(\boldsymbol{x}_{j b}, \boldsymbol{y}_{j b}, \boldsymbol{z}_{j b}\right)$ coordinate system as:

$$
{ }^{j b} \boldsymbol{g}_{j, k}=\left[-T \phi_{j b} C \psi_{j b}\left|t_{j, k}\right|, t_{j, k}, \delta_{j b} T \phi_{j b} S \psi_{j b}\left|t_{j, k}\right|\right]^{\mathrm{T}}
$$

where $\phi_{j b}$ and $\psi_{j b}$ are the pressure angle and pitch cone angle of the output-end gear of link $j$, $T \phi_{j b}=\tan \left(\phi_{j b}\right), t_{j, k}$ is the tangential component of $\boldsymbol{g}_{j, k},\left|t_{j, k}\right|$ is the absolute value of $t_{j, k}$, and $\delta_{j b}=1$ if the cone apex of the output-end gear of link $j$ falls on the negative side of $z_{j}$, otherwise $\delta_{j b}=-1$.

Note that the radial and axial components of the gear contact force serve no useful purpose as far as the transmission of torque is concerned. Hence, the tangential component of the contact force, $t_{j, k}$, is defined as the input transmitted force of link $k$. In Eq. (21), it can be seen that $t_{j, k}$ is the only unknown, since $\phi_{j b}, \psi_{j b}$, and $\delta_{j b}$ are geometry characteristics of gears. With the transformation matrix shown in Eq. (20) and rotation matrices shown in Eqs. (1) and (8), gear contact forces can be expressed in a preferred coordinate system.

For the example mechanism shown in Fig. $4,{ }^{1} \boldsymbol{H}_{2 a}$ and ${ }^{2} \boldsymbol{H}_{7 b}$ can be written as:

$$
{ }^{1} \boldsymbol{H}_{2 a}=\left[\begin{array}{lll}
0 & 1 & 0 \\
-1 & 0 & 0 \\
0 & 0 & 1
\end{array}\right] \text { and }{ }^{2} \boldsymbol{H}_{7 b}=\left[\begin{array}{lll}
0 & 0 & 1 \\
0 & 1 & 0 \\
-1 & 0 & 0
\end{array}\right]
$$

The gear contact force $\boldsymbol{g}_{6,7}$ and $\boldsymbol{g}_{4,1}$ are:

$$
{ }^{6 b} \boldsymbol{g}_{6,7}=\left[-T \phi_{6 b} C \psi_{6 b}\left|t_{6,7}\right|, t_{6,7},-T \phi_{6 b} S \psi_{6 b}\left|t_{6,7}\right|\right]^{\mathrm{T}}
$$

and

$$
{ }^{4 b} \boldsymbol{g}_{4,1}=\left[-T \phi_{4 b}\left|t_{4,1}\right|, t_{4,1}, 0\right]^{\mathrm{T}}
$$




\section{Dynamic analysis}

The force and moment balance equations of an individual link are the Newton and Euler's equations. It is possible to establish balance equations for each link and solved for all the unknowns simultaneously. However, this would be computational intensive and inefficient. If there is fewer equation(s) involved in solving the input transmitted force of a link, then this set of equation(s) can be solved independently. Once the input transmitted force of a link is solved, the input transmitted force of the adjacent links along the heavy-edge path of the MTL may become solvable from the concept of torque transmission. In what follows, a two-step methodology for the dynamic analysis of GRMs with pseudo- triangular structure will be presented and then extended to GRMs with general structure form.

\subsection{Moment balance along rotational axis}

The input transmitted force of a link can be solved from the moment balance equation with respect to a point along its rotational axis. Fig. 5 shows the free body diagram of a typical primary link $i$. Assume that the primary link $i$ carries a secondary link $k$ and is meshed with a secondary link $j$. The moment balance equation of link $i$ with respect to $o_{i-1}$ along $z_{i-1}$ can be written as:

$$
\begin{aligned}
\lambda_{i a} \cdot t_{j, i}= & \left.\left.\left\{\boldsymbol{n}_{i+1, i}\right)_{z}+\left[\left(\boldsymbol{b}_{i+1, i}+\boldsymbol{p}_{i, i-1}\right) \times \boldsymbol{f}_{i+1, i}\right]_{z}\right\}+\boldsymbol{n}_{k, i}\right)_{z}+\left[\left(\boldsymbol{b}_{k, i}+\boldsymbol{p}_{i, i-1}\right)\right. \\
& \left.\left.\times \boldsymbol{f}_{k, i}\right]_{z}+\boldsymbol{N}_{i}^{*}\right)_{z}+\left[\left(\boldsymbol{r}_{i c}+\boldsymbol{p}_{i, i-1}\right) \times \boldsymbol{F}_{i}^{*}\right]_{z}
\end{aligned}
$$

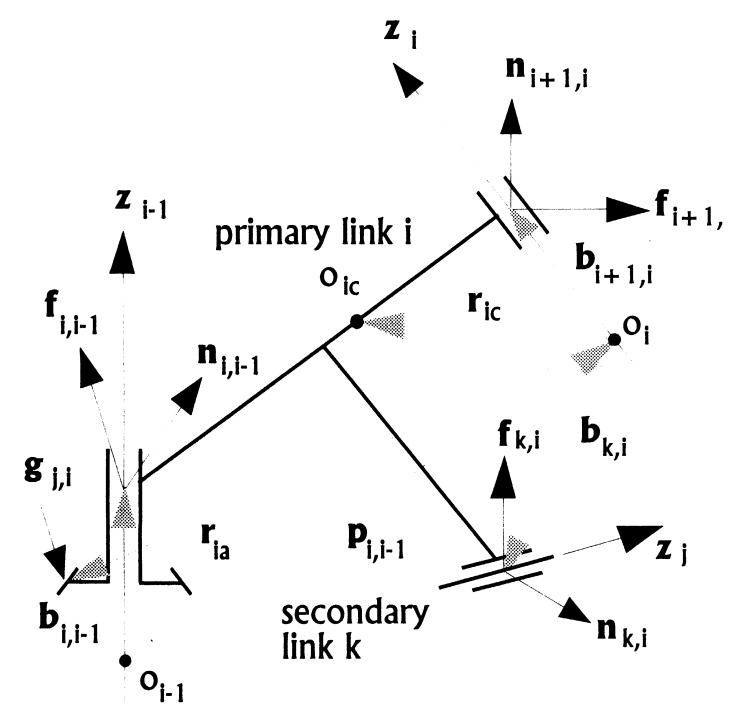

Fig. 5. Free body diagram of a typical primary link. 
where $\lambda_{i a}$ is the radius of input-side gear of link $i, b_{j, i}$ is the position vector from $o_{i}$ to the location of joint $(i, j), \boldsymbol{r}_{i c}$ is the position vector from $o_{i}$ to mass center of link $i$ and $t_{j, i}$ is the input transmitted force of primary link $i$ and the vectors enclosed by \{\} are defined as the effective external forces of primary link $i$.

For the primary link $i$ to be the end link of MTL of the highest level, Eq. (25) can be reduced to the following since the end-effector carries no secondary link.

$$
\left.\left.\lambda_{i a} \cdot t_{j, i}=\left\{\boldsymbol{n}_{\mathrm{ext}, i}\right)_{z}+\left[\left(\boldsymbol{b}_{\mathrm{ext}, i}+\boldsymbol{p}_{i, i-1}\right) \times \boldsymbol{f}_{\mathrm{ext}, i}\right]_{z}\right\}+\boldsymbol{N}_{i}^{*}\right)_{z}+\left[\left(\boldsymbol{r}_{i c}+\boldsymbol{p}_{i, i-1}\right) \times \boldsymbol{F}_{i}^{*}\right]_{z}
$$

where $\boldsymbol{n}_{\mathrm{ext}, i}$ and $\boldsymbol{f}_{\mathrm{ext}, i}$ are the external force and moment of the GRM.

Hence, the input transmitted force of the end-effector, $t_{j, i}$, is the only unknown in Eq. (26) and can be evaluated with the external forces given at a specified motion status.

Fig. 6 shows the free body diagram of a typical secondary link $j$ which meshed with secondary links and $j+1$ at its input-side and output-side, respectively, and carried by primary link $i-1$. The moment balance equation of link $j$ with respect to $o_{j}$ along $z_{j}$ can be written as:

$$
\left.\lambda_{j a} \cdot t_{j-1, j}=N_{j}^{*}\right)_{z}+\lambda_{j b} \cdot t_{j, j+1}
$$

where $\lambda_{j b}$ is the radius of output-side gear of link $j$.

For the secondary link $j$ to be the last secondary link of MTL of the highest level, Eq. (27) can be reduced to the following since the last secondary link $j$ is meshed with end primary link $i$.

$$
\left.\lambda_{j a} \cdot t_{j-1, j}=N_{j}^{*}\right)_{z}+\lambda_{j b} \cdot t_{j, i}
$$

From Eq. (28), it can be seen that the input transmitted force of secondary link $j, t_{j-1, j}$, is the only unknown and can be evaluated when the input transmitted force of link $i, t_{j, i}$, is determined from Eq. (26) at a specified motion status. The procedure can continue throughout the secondary links of the MTL of highest level. Thus, the actuator torque associated with the highest level MTL can be computed by evaluating the moment balance equation along the rotational axis of links starting from the end primary link followed by the secondary links backward along the heavy-

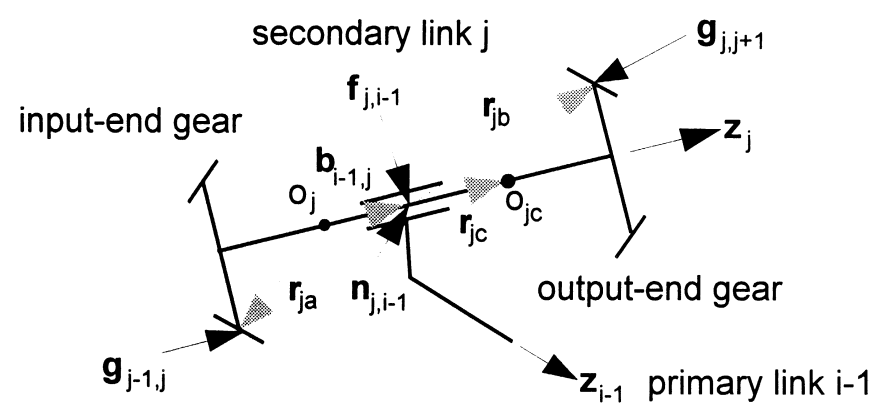

Fig. 6. Free body diagram of a typical secondary link. 
edged path of the MTL. Note that for secondary link $j$ to be the input link, Eq. (27) reduces to

$$
\left.\xi_{j}=N_{j}^{*}\right)_{z}+\lambda_{b} \cdot t_{j, j+1}
$$

where $\xi_{j}$ is the actuator torque applied at input link $j$.

For GRMs with pseudo-triangular structure matrix, the computation process repeats itself for the primary link and secondary links of the next level MTL, with the determined effective external forces, until all the required actuator torques to maintain the specified motion status are solved. Thus, the dynamic equations of motion can be established efficiently and systematically without solving all the reaction forces.

Referring to the canonical graph representation of example mechanism shown in Fig. 2(b), there are three MTLs connected to the three primary links, and link 3 is the end effector. The evaluation procedure of the input transmitted force follows the following sequence $[(3 \rightarrow 7 \rightarrow 6),(2 \rightarrow 5)$, and $(1 \rightarrow 4)]$. Table 1 shows the effective external forces of each MTL to be evaluated before the computation. From Table 1, it can be seen that there are only 11 unknowns to be evaluated to establish the dynamic equations of the example GRM.

\subsection{Balance equations of links}

Referring to Fig. 5, the force and moment balance equations for the primary link $i$ with respect to $o_{i-1}$ can be written as:

$$
\boldsymbol{f}_{i, i-1}-\boldsymbol{g}_{j, i}=\boldsymbol{F}_{i}^{*}+\boldsymbol{f}_{i+1, i}+\boldsymbol{f}_{k, i}
$$

and

$$
\begin{aligned}
& \boldsymbol{n}_{i, i-1}+\boldsymbol{b}_{i, i-1} \times \boldsymbol{f}_{i, i-1}-\left(\boldsymbol{r}_{i a}+\boldsymbol{p}_{i, i-1}\right) \times \boldsymbol{g}_{j, i} \\
& =\boldsymbol{N}_{i}^{*}+\left(\boldsymbol{r}_{i c}+\boldsymbol{p}_{i, i-1}\right) \times \boldsymbol{F}_{i}^{*}+\boldsymbol{n}_{i+1, i}+\left(\boldsymbol{b}_{i+1, i}+\boldsymbol{p}_{i, i-1}\right) \times \boldsymbol{f}_{i+1, i}+\boldsymbol{n}_{k, i}+\left(\boldsymbol{b}_{k, i}+\boldsymbol{p}_{i, i-1}\right) \\
& \quad \times \boldsymbol{f}_{k, i}
\end{aligned}
$$

where $\boldsymbol{r}_{i a}$ is the position vector from $o_{i}$ to the meshed point of input-end gear of link $i$. Referring to Fig. 6, the force and moment balance equations for the secondary link $j$ with

Table 1

Position vectors of the example GRM

\begin{tabular}{llll}
\hline Level of MTL & Links & Effective external force & Input transmitted forces \\
\hline 3 & 3 & $f_{\text {ext }, 3}, n_{\text {ext }, 3}$ & $t_{7,3}$ \\
& 7 & & $t_{6,7}$ \\
2 & 6 & $\left(f_{3}, 2\right)_{y},\left(f_{7}, 2\right)_{y}$ & $\xi_{6}$ \\
& 2 & $\left(f_{2}, 1\right)_{x},\left(f_{3}, 2\right)_{y},\left(f_{6}, 1\right)_{x}$ & $t_{5,2}$ \\
1 & 5 & & $\xi_{5,1}$ \\
& 1 & & $\xi_{4}$ \\
\hline
\end{tabular}


respect to $o_{j}$ can be written as:

$$
\boldsymbol{f}_{j, i-1}-\boldsymbol{g}_{j-1, j}=\boldsymbol{F}_{j}^{*}-\boldsymbol{g}_{j, j+1}
$$

and

$$
\boldsymbol{n}_{j, i-1}-\boldsymbol{r}_{j a} \times \boldsymbol{g}_{j-1, j}+\boldsymbol{b}_{i-1, j} \times \boldsymbol{f}_{j, i-1}=\boldsymbol{N}_{j}^{*}-\boldsymbol{r}_{j b} \times \boldsymbol{g}_{j, j+1}+\boldsymbol{r}_{j c} \times \boldsymbol{F}_{j}^{*}
$$

where $\boldsymbol{r}_{j b}$ is the position vector from $o_{j}$ to the meshed point of output-end gear of link $i$.

$$
\begin{aligned}
& { }^{j} \boldsymbol{r}_{j a}={ }^{j} \boldsymbol{p}_{j a, j}+{ }^{j} \boldsymbol{R}_{i-1}{ }^{i-1} \boldsymbol{H}_{j a}{ }^{j a} \boldsymbol{u}_{j a} \\
& { }^{j} \boldsymbol{b}_{i-1, j}={ }^{j} \boldsymbol{R}_{i-1}{ }^{i-1} \boldsymbol{b}_{j, i-1}-{ }^{j} \boldsymbol{P}_{j, i-1}
\end{aligned}
$$

and

$$
{ }^{j} \boldsymbol{r}_{j b}={ }^{j} \boldsymbol{p}_{j b, j}+{ }^{j} \boldsymbol{R}_{i-1}{ }^{i-1} \boldsymbol{H}_{j b}{ }^{j b} \boldsymbol{u}_{j b} \quad \text { if link } i-1 \text { is carrier of gear pair }(j, j+1)
$$

or

$$
{ }^{j} \boldsymbol{r}_{j b}={ }^{j} \boldsymbol{p}_{j b, j}+{ }^{j} \boldsymbol{R}_{i-1}{ }^{i-1} \boldsymbol{R}_{i}^{i} \boldsymbol{H}_{j b}{ }^{j b} \boldsymbol{u}_{j b} \quad \text { if link } i \text { is carrier of gear pair }(j, j+1)
$$

and where $\boldsymbol{u}_{j a}=\left[\lambda_{j a}, 0,0\right]^{\mathrm{T}}$ is the position vector from the input-end gear center $o_{j a}$ of link $j$ to the meshed point and $\boldsymbol{u}_{j b}=\left[\lambda_{j b}, 0,0\right]^{\mathrm{T}}$ is the position vector from the output-end gear center $o_{j b}$ of link $j$ to the meshed point.

Hence, the remaining unsolved reaction forces are evaluated link-by-link backward along the heavy-edged paths from the highest level MTL to the lower level ones. Since there are fewer linear equations and fewer unknowns to be solved simultaneously, this step procedure can be computational efficient. Referring to Figs. 3 and 4, the position vectors required for the evaluation of Eqs. (25)-(33) are ${ }^{1} \boldsymbol{r}_{1 c}=[0,-s 1,0]^{\mathrm{T}},{ }^{2} \boldsymbol{r}_{2 c}=\left[-s_{2}, 0,0\right]^{\mathrm{T}},{ }^{7} \boldsymbol{r}_{7 c}=\left[0,0,-s_{7}\right]^{\mathrm{T}},{ }^{2} \boldsymbol{b}_{7}, 2=$ $\left.\left[-s_{2}, 0,0\right]^{\mathrm{T}},{ }^{6} \boldsymbol{b}_{1}, 6=[0,0, b]\right]^{\mathrm{T}}$ and ${ }^{3} \boldsymbol{r}_{3 c}={ }^{4} \boldsymbol{r}_{4 c}={ }^{5} \boldsymbol{r}_{5 c}={ }^{6} \boldsymbol{r}_{6 c}={ }^{3} \boldsymbol{b}_{\mathrm{ext}}, 3={ }^{4} \boldsymbol{b}_{0}, 4={ }^{5} \boldsymbol{b}_{0}, 5=[0,0,0]^{\mathrm{T}}$. The evaluation procedure of remaining unsolved reaction force listed in Table 2 , follows the following sequence $[(3 \rightarrow 7 \rightarrow 6),(2 \rightarrow 5)$, and $(1 \rightarrow 4)]$.

Table 2

The effective external forces and input transmitted forces

\begin{tabular}{lll}
\hline Level of MTL & Links & Unknowns \\
\hline 3 & 3 & $\left(\boldsymbol{f}_{3}, 2\right)_{x},\left(\boldsymbol{f}_{3}, 2\right)_{z},\left(\boldsymbol{n}_{3}, 2\right)_{x},\left(\boldsymbol{n}_{3}, 2\right)_{y}$ \\
& 7 & $\left(\boldsymbol{f}_{7}, 2\right)_{x},\left(\boldsymbol{f}_{7}, 2\right)_{z},\left(\boldsymbol{n}_{7}, 2\right)_{x},\left(\boldsymbol{n}_{7}, 2\right)_{y}$ \\
2 & 6 & $\left(\boldsymbol{f}_{6}, 1\right)_{y},\left(\boldsymbol{f}_{6}, 1\right)_{z},\left(\boldsymbol{n}_{6}, 1\right)_{y},\left(\boldsymbol{n}_{6}, 1\right)_{x}$ \\
& 2 & $\left(\boldsymbol{f}_{2}, 1\right)_{y},\left(\boldsymbol{f}_{2}, 1\right)_{z},\left(\boldsymbol{n}_{2}, 1\right)_{y},\left(\boldsymbol{n}_{2}, 1\right)_{x}$ \\
1 & 5 & $\left(\boldsymbol{f}_{5}, 0\right)_{x},\left(\boldsymbol{f}_{5}, 0\right)_{y},\left(\boldsymbol{f}_{5}, 0\right)_{z},\left(\boldsymbol{n}_{5}, 0\right)_{x},\left(\boldsymbol{n}_{5}, 0\right)_{y}$ \\
& 1 & $\left(\boldsymbol{f}_{1}, 0\right)_{x},\left(\boldsymbol{f}_{1}, 0\right)_{y},\left(\boldsymbol{f}_{1}, 0\right)_{z},\left(\boldsymbol{n}_{1}, 0\right)_{x},\left(\boldsymbol{n}_{1}, 0\right)_{y}$ \\
& 4 & $\left(\boldsymbol{f}_{4}, 0\right)_{x},\left(\boldsymbol{f}_{4}, 0\right)_{y},\left(\boldsymbol{f}_{4}, 0\right)_{z},\left(\boldsymbol{n}_{4}, 0\right)_{x},\left(\boldsymbol{n}_{4}, 0\right)_{y}$ \\
\hline
\end{tabular}




\section{Mechanisms with general structure matrix}

Fig. 7(a) shows the Bendix wrist mechanism and Fig. 7(b) is its canonical graph. There are two MTLs connected to link 3, while no MTL connected to link 2. The moment balance equation of primary link 3 with respect to $o_{2}$ along $z_{2}$ can be obtained by modifying Eq. (25) as:

$$
\left.\left.\lambda_{3 a} \cdot t_{8,3}+\lambda_{3 a} \cdot t_{6,3}=\boldsymbol{n}_{\mathrm{ext}, 3}\right)_{z}+\left[\left(\boldsymbol{b}_{\mathrm{ext}, 3}+\boldsymbol{p}_{3,2}\right) \times \boldsymbol{f}_{\mathrm{ext}, 3}\right]_{z}+\boldsymbol{N}_{3}^{*}\right)_{z}+\left(\left(\boldsymbol{r}_{3 c}+\boldsymbol{p}_{3,2}\right) \times \boldsymbol{F}_{3}^{*}\right)_{z}
$$

The moment balance equation of primary link 2 with respect to $o_{1}$ along $z_{1}$ can be modified from Eq. (25) as:

$$
\left.\left.0=n_{3,2}\right)_{z}+\left[\left(\boldsymbol{b}_{3,2}+\boldsymbol{p}_{2,1}\right) \times \boldsymbol{f}_{3,2}\right]_{z}+\boldsymbol{N}_{2}^{*}\right)_{z}+\left(\left(\boldsymbol{r}_{2 c}+\boldsymbol{p}_{2,1}\right) \times \boldsymbol{F}_{2}^{*}\right)_{z}
$$

The force balance equation about link 2, by Eq. (30), can be written as:

$$
\boldsymbol{f}_{3,2}=\boldsymbol{F}_{3}^{*}+\boldsymbol{f}_{\mathrm{ext}, 3}+\boldsymbol{g}_{8,3}+\boldsymbol{g}_{6,3}
$$

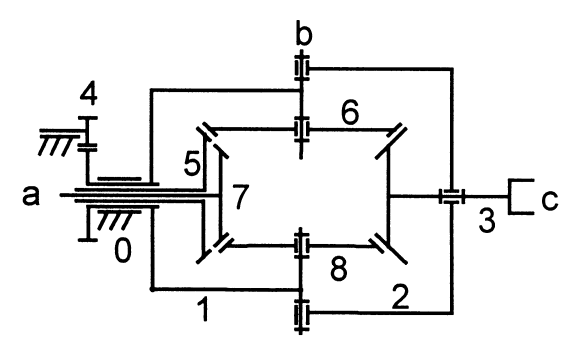

(a)

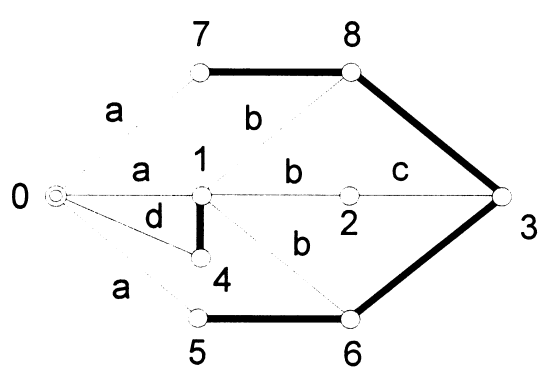

(b)

Fig. 7. (a) Bendix wrist mechanism. (b) Canonical graph of Bendix wrist mechanism. 
From Eq. (39), it can be seen that the effective external force of link 2, $\boldsymbol{f}_{3,2}$, can be expressed in terms of the unknown input transmitted forces $t_{8,3}$ and $t_{6,3}$ which are tangential components of $\boldsymbol{g}_{8,3}$ and $\boldsymbol{g}_{6,3}$. Hence, the two unknown input transmitted forces $t_{8,3}$ and $t_{6,3}$ can be solved with Eqs. (37) and (38). The input torques of the mechanism can then be evaluated in the sequence: $(3 \rightarrow 2) \rightarrow 8 \rightarrow 7 \rightarrow 6 \rightarrow 5$, and $(1 \rightarrow 4)$. With the solved input transmitted forces of links, the unknown dynamic loads at revolute joints are evaluated by the same procedure using balance equations of links as stated above.

\section{Summary}

In this paper, a two-step method for the inverse dynamic analysis of a general class of GRMs is developed. The computation begins from the highest level MTL towards the lowest level MTL. Input transmitted forces are computed from the moment balance equation along axes of rotation starting from the end primary link backward along the heavy-edged path in an MTL. Dynamic equations are derived from the computed input transmitted forces. With the solved input transmitted forces, the remaining unknown reaction forces at revolute joints can then be evaluated efficiently from the balance equations of links. Thus, the inverse dynamic problem are solved systematically without the need of solving the entire system of equation simultaneously.

\section{References}

[1] MDI, User's Guide to ADAMS, Mechanical Dynamics, Inc. 5th ed., Ann Arbor, MI, 1981.

[2] CADSI, DADS User's Manual Rev. 5.0, Computer Aided Design Software Inc. Oakland, Iowa, 1988.

[3] J.J. Uicker, Ph.D. Dissertation. Northwestern University, Evanston, III, 1965.

[4] J.M. Hollerbach, IEEE Trans., Systems, Man, Cybernetics SMC-10 (11) (1980) 730-736.

[5] W.M. Armstrong, Proc. 5th World Congr., Machines and Mechanisms Theory, vol. 2, 1979, pp. $1343-1346$.

[6] D.E. Orin, R.B. McGhee, M. Vukobratovic, G. Hartoch, Math. Biosci 43 (1979) 107-130.

[7] J.Y.S. Luh, M.W. Walker, R.P. Paul, ASME Trans, Journal of Dynamic Systems, Measurements and Control 120 (1980) 69-76.

[8] M.W. Walker, D.E. Orin, ASME J. of Dynamic System, Measurement, and Control 104 (1982) $205-211$.

[9] C.S.G. Lee, B.H. Lee, R. Nigam, in: Proc. 22nd Conf. Decision and Control, San Antonio, TX, 1983, pp. 1205-1210.

[10] L.W. Tsai, D.Z. Chen, T.W. Lin, ASME J. of Mechanical Design 120 (1998) 240-244.

[11] L.W. Tsai, IEEE Journal of Robotics and Automation 4 (2) (1988) 150-155.

[12] G. Chartrand, O.R. Oellermann, Applied and Algorithmic Graph Theory. McGraw-Hill, New York, 1993.

[13] D. Chen, Lung-Wen. Tsai, J. of Applied Mechanisms and Robotics 1 (3) (1994) 17-25.

[14] J. Denavit, R.S. Hartenberg, ASME J. of Applied Mechanics 77 (1955) 215-221.

[15] S.L. Chang, L.W. Tsai, IEEE Trans on Robotics and Automation 6 (1) (1990) 97-103. 